\title{
C̆ERENKOV WAVEGUIDE SHG: A LESS CRITICAL WAY TO REACH THE EFFICIENCY PEAK
}

\author{
L. KotaC̆KA*, J. С̆TYRoKÝ
}

Institute of Radio Engineering and Electronics

Chaberská 57, 18251 Praha 8, Czech Republic

AND H.J.W.M. HoEKSTRA

Light Device Group, University of Twente

P.O. Box 217, 7500 AE Enschede, Netherlands

\begin{abstract}
It is well known that the conversion efficiency of the SHG in the Cerenkov regime in a wa veguide with a nonlinear substrate exhibits a very sharp peak. Its position in a "pump wavelength-guide thickness" diagram follows a modified dispersion relation. A particular material combination exactly determines both the pump wavelength and the guide thickness. The existence of the peak was also experimentally proved with relatively high normalised conversion efficiency of $210 \% /(\mathrm{cm} \mathrm{W)}$. However, in order to achieve such a high conversion efficiency, the required tolerance in the guide thickness should be smaller than $0.5 \mathrm{~nm}$ (the FWHM of the peak is approximately $0.8 \mathrm{~nm}$ ). The allowed variations in refractive indices are also very low $\left(<10^{-3}\right)$. The recent theoretical study of the authors showed that an additional layer (idler) inserted into the waveguide facilitates more degrees of freedom which could relax the conditions for reaching the efficiency peak. This paper presents a detailed study of the behaviour of four-layer waveguide devices with a nonlinear substrate which promise a less constrained way to reach the efficiency peak.
\end{abstract}

PACS numbers: 42.65.Ky, 42.82.Et

\section{Introduction}

The optical second harmonic generation (SHG) phenomena in diverse configurations of nonlinear waveguides have already been extensively studied since $1970 \mathrm{~s}$ (see e.g. [1-3]). However, some issues, especially those concerning the transition

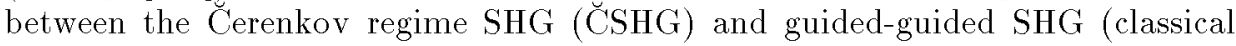

* corresponding author; e-mail: kotacka@ure.cas.cz 
phase-matching), still attract attention $[4,5]$ because they have not been well understood yet. A major part of the research efforts have been devoted during last years, of course, to the optimisation of the guided-guided interaction (GSHG). The common objective of those efforts has been to obtain as high second harmonic conversion efficiency as possible. It is well known (see e.g. [2]) that the second harmonic power generated through the guided mode in the GSHG arrangement increases with the squared interaction length $\left(P_{2 \omega} \propto L^{2}\right)$, while that one in the $\breve{C}$ erenkov arrangement obeys only the linear dependence $\left(P_{2 \omega} \propto L\right)$. Since both SHG arrangements follow the quadratic dependence on the pump radiation power $\left(P_{2 \omega} \propto P_{\omega}^{2}\right)$, the GSHG offers higher generated power except of extremely short propagation lengths.

However, Asai et al. [6] reported in 1992 the existence of the sharp peak in the conversion efficiency maximum in the Čerenkov regime close to the cut-off of the guided second harmonic mode. They also noted that the conventional theory [3] describing the Cerenkov conversion efficiency diverges at this point and at its very close vicinity. They further stated that the expression for the conversion efficiency is to a high degree accurate in the range of the Cerenkov angle (see below) down to 0.1 degree. It means, as will be seen later, that the theory is valid only in that region where the generated power is proportional to the propagation distance. Two papers of Doumuki et al. [7,8] reported an experimental proof of the existence of the peaked conversion efficiency of the C̆SHG with surprisingly high conversion efficiency. Moreover, they recognised two principal characteristics of the peak. Namely, the peak is quite difficult to reach because the material requirements impose the accuracy of the guide thickness of about $0.2 \mathrm{~nm}$ and the same stringent constraint holds for the accuracy $\left(<10^{-3}\right)$ of all refractive indices in the waveguide. Further, the peak is situated just at the transition between the Čerenkov regime and the guided-guided one. The most important observation was that the peaked efficiency continuously passed into the "ridge" (the region of the perfect phase-matching) created by a squared sinc function in the expression giving the conversion efficiency for GSHG (see e.g. equation (43) in [2]). In 1998 Chang and Shaw [9] shed light on the origin of the condition (equation (11) therein) yielding the peak position. Authors' recent message [10] generally explained the existence of the peak condition and the equation yielding the peak position was given there as well (see equation (4) therein). That message also studied in particular the design of the waveguide structure which would, by means of the Čerenkov peak, give high conversion efficiency for a broad interval of pump wavelengths.

Until the publication of paper [4], the theoretical value of the conversion efficiency had not been known. That paper, for the first time, derived a novel theory for small Cerenkov angles and showed that for Črenkov angles approaching zero, the conversion efficiency is proportional to the $3 / 2$-th power of the interaction length. Moreover, the follow-up paper [5] proved that the theory held for the TM case as well and described the comparison between TE and TM SHG conversions.

The aim of this paper is to further analyse how the material composition of a waveguide influences the peak position. The theoretical study of highly efficient C̆SHG in waveguides with a nonlinear substrate has revealed that an additional layer ( $i d l e r$ ) of certain parameters relaxes the conditions for reaching the efficiency 
peak. A previously fixed peak position (given by a material composition) may shift as a consequence of an idler presence in the waveguide.

The structure containing the idler layer of a refractive index slightly above the required value of the effective index seems to be very promising. The structure with an idler layer of the thickness of about 300 or $500 \mathrm{~nm}$ exhibits lower sensitivity of the efficiency peak to the fluctuations of the pump wavelength changes. Finally, a detailed study of the overlap integral - an important quantity for the conversion efficiency - did not reveal any important declination compared to that of a three-layer waveguide.

\section{Fundamentals of waveguide SHG theory}

For the sake of brevity, we shall deal only with the $\mathrm{TE}^{\omega}-\mathrm{TE}^{2 \omega}$ conversion without any loss of generality. We shall further limit ourselves to studying the first possible second harmonic interaction between the 0th mode at the fundamental frequency $\omega$ and the 1 st mode at the second harmonic frequency $2 \omega$. The structure of a general four-layer waveguide is shown in Fig. 1. According to equation (38) in [4], the conversion efficiency for large Cerenkov angles under non-depleted pump approximation (NDPA) is

$$
P_{2 \omega}=\eta_{c} L P_{\omega}^{2}
$$

with the normalised conversion efficiency given by

$$
\eta_{c} \approx 8 k_{2 \omega}^{2} \frac{Z_{0}}{N^{3}} d_{\mathrm{eff}}^{2} F^{2} \cot \theta_{c},
$$

where $k_{2 \omega}=2 k_{\omega}=2 \omega / c$ ( $c$ being the velocity of light in vacuum), $Z_{0}=\sqrt{\mu_{0} / \varepsilon_{0}}$ is the free-space impendance, $N$ is the effective refractive index of the pump guided

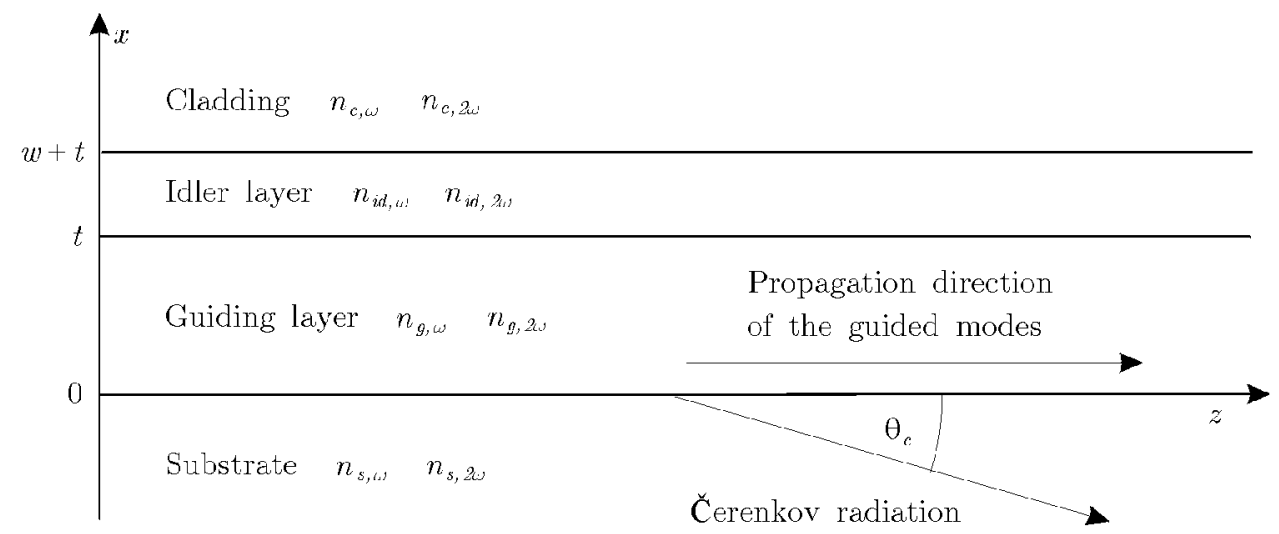

Fig. 1. The scheme of the waveguide under study consisting of four layers. Each layer is characterised by wavelength depending refractive index (assumed thus for the both frequencies $\omega, 2 \omega), t$ is the thickness of the guiding layer, while $w$ is the thickness of the additional (idler) layer. Cerenkov second harmonic radiation escapes into the substrate under the Cerenkov angle $\theta_{c}$. 
mode, $d_{\mathrm{eff}}$ is the pertinent effective nonlinear constant (i.e. namely $d_{33}$ in case of KTP). Finally, $F$ is the so-called overlap integral defined as (for detailed description see [4])

$$
F=\int_{-\infty}^{0} e_{2 \omega}^{2}(x) e_{\omega}(x) \mathrm{d} x,
$$

where $\epsilon_{\Omega}(x)(\Omega=\omega, 2 \omega)$ are the field components in the substrate. The expression (1) is valid for the Cerenkov angles larger than approximately $0.1^{\circ}$. For the angles below this value, where Eq. (1) ceases to be valid, we have derived the following expression:

$$
P_{2 \omega, \theta \rightarrow 0}=\eta_{p} L^{3 / 2} P_{\omega}^{2}
$$

As before in Eq. (1), (2), the normalisation constant is approximately given by

$$
\eta_{p} \approx \frac{16}{3 \sqrt{\pi}} \frac{k_{2 \omega}^{5 / 2}}{N^{3}} d_{\text {eff }}^{2} F^{2} .
$$

Further, we introduce the maximal value of the renormalised (renormalised to $1 \mathrm{~mm}$ of the interaction length) conversion efficiency $\eta_{\max }$ at the top of the peak, which is approximately equal to (see Fig. 7 in [4])

$$
\eta_{\max } \approx 0.06 \mathrm{~W}^{-1} \text {. }
$$

The highest theoretical value of the generated power then obeys the following simple approximate relation (we shall implicitly assume here that this value is approximately valid apart from a material composition of the waveguide):

$$
P_{2 \omega, \text { peak }} \approx 0.06\left(\frac{L}{L_{1 \mathrm{~mm}}}\right)^{3 / 2} P_{\omega}^{2} .
$$

The bracketed term denotes the relative interaction length related to $1 \mathrm{~mm}$. For example, assuming the pump power of $100 \mathrm{~mW}$ (confined to $1 \mu \mathrm{m}$ - see the notation in [4]) we get about $0.6 \mathrm{~mW}, 6.7 \mathrm{~mW}, 11.1 \mathrm{~mW}$, and $31.6 \mathrm{~mW}$ for the interaction lengths $1 \mathrm{~mm}, 5 \mathrm{~mm}, 7 \mathrm{~mm}$, and $10 \mathrm{~mm}$, respectively. Note that the value of $31 \mathrm{~mW}$ is obviously out of NDPA. The other values are in a generally good agreement with Doumuki's experiments $[7,8]$. At this place, we shall remind that Doumuki introduced in $[7,8]$ the normalised efficiency in $[\% /(\mathrm{cm} \mathrm{W})]$, which just may express a value of the conversion efficiency at the top of the peak, but it does not have any physical meaning, because of the linear dependence on the interaction length.

The above given theoretical values of possibly obtainable second harmonic power in the Čerenkov regime are in a remarkable agreement with those reported for the guided-guided regime [8] when the interaction length is several $\mathrm{mm}$ (say until $L<5 \mathrm{~mm}$ ). Moreover, the C̆ SHG gives essentially higher conversion for very short interaction lengths. These conclusions clearly justify the importance of further research into the study of the SHG conversion efficiency at the vicinity of the peak. Finally, we should also note that the light generated in the Čerenkov regime spreads mainly through the substrate, which may be thus understood as a bulk medium in the form of the plane wave. Since the propagation losses of guided waves are approximately function of $\lambda^{-4}$, the C̆SHG configuration offers an essential advantage compared to GSHG. 
The following section deals with the peak position which is, in fact, exactly given for a certain material composition. Hence, we shall try to find a solution providing a higher degree of freedom in the tuning of the peak position by changing the pump wavelength.

\section{Condition for the peak}

The detailed study of the origin of the peak was done for the first time in $[9,10]$ and further considered in $[4,5]$. Although those studies were performed for a three-layer waveguide, the obtained results are valid, in general, for multilayer waveguides as well. We shall thus briefly present a short summary of those results.

As it was shown in $[9,10]$, the peaked conversion efficiency occurs when the Cerenkov angle approaches zero degree, i.e. the peak is situated at the boundary of the Cerenkov regime. It is well known [3-10] that this boundary is defined by the following identity between the effective refractive index of the guided mode at the fundamental frequency and the refractive index of the substrate of the second harmonic frequency:

$$
N=n_{\mathrm{s}, 2 \omega} \text {. }
$$

On the other hand, from the point of view of the guided second harmonic mode, the peak occurs at the cut-off of this guided mode. Hence, the position of the peak can be found solving the following modified dispersion equation, which consist of the dispersion equations for both fundamental and second harmonic guided modes by putting the effective refractive indices of the both modes equal to $\eta_{\mathrm{s}, 2 \omega}$ to fulfil the conditions given above. This modified dispersion equation can be written in the form (according to the basic dispersion equation for a four-layer waveguide given in [11], equation (5.39) therein)

$$
\frac{\arctan \left(u_{\mathrm{s}} / \mathrm{i} u_{\mathrm{g}}\right)+\arctan \phi}{u_{\mathrm{g}}}-\frac{\arctan \Phi+\pi}{U_{\mathrm{g}}}=0,
$$

where

$$
\phi=\left(\frac{u_{\mathrm{id}}}{u_{\mathrm{g}}}\right) \tan \left[-u_{\mathrm{id}} w+\arctan \left(u_{\mathrm{c}} / \mathrm{i} u_{\mathrm{id}}\right)\right]
$$

and similarly

$$
\Phi=\left(\frac{U_{\mathrm{id}}}{U_{\mathrm{g}}}\right) \tan \left[-U_{\mathrm{id}} w+\arctan \left(U_{\mathrm{c}} / \mathrm{i} U_{\mathrm{id}}\right)\right]
$$

with $u_{i}=k_{\omega} \sqrt{n_{i, \omega}^{2}-n_{\mathrm{s}, 2 \omega}^{2}}$ and $U_{i}=k_{2 \omega} \sqrt{n_{i, 2 \omega}^{2}-n_{\mathrm{s}, 2 \omega}^{2}}$. The subscripts $i=\mathrm{s}$, $\mathrm{g}$, id, c denote substrate, guiding layer, idler layer, and cladding, respectively. Of course, Eq. (9) is nothing else than the relation obeying the equality, which must be satisfied between the thickness expressed through the guided fundamental mode (under the condition (8)) and that one expressed through the guided second harmonic mode at the cut-off. The solution of (9) gives the pump wavelength which may be just converted by the highest conversion efficiency, in the other words converted by the peak. (Remind that the effective indices are generally given by the Sellmeier dispersion formula (see e.g. [12], p. 97), i.e. the refractive 
indices depend on the wavelength and thus in general $u_{i}=u_{i}(\lambda), U_{i}=U_{i}(\lambda)$.) The thickness of the guiding layer is then, of course, simply determined either from the dispersion relation of the guided fundamental mode or from the guided second harmonic mode at the cut-off. Namely (choosing the fundamental guided mode)

$$
t=\frac{\arctan \left(u_{\mathrm{s}} / \mathrm{i} u_{\mathrm{g}}\right)+\arctan \phi}{u_{\mathrm{g}}} .
$$

We shall further introduce a "thickness-lambda" diagram. The pair "guide thickness-pump wavelength" coming from the relations (9) and (10) makes the coordinates of the peak position in that diagram for a given structure. In the next paragraph, we discuss the positions of the peak for waveguides of various material configurations.

\section{Discussion of various configurations}

We start with the study of the four-layer waveguide consisting of silicon based materials. Namely, the guiding layer consists of the PECVD $\mathrm{Si}_{3} \mathrm{~N}_{4}(n \approx 2)$. The cladding is considered to be composed either of $\mathrm{SiO}_{2}(n \approx 1.46)$ or $\mathrm{SiON}$ $(n \approx 1.73)$ or air

Note: The digits in the following eight diagrams indicate the thickness $w$ of a pertinent idler in nanometers.

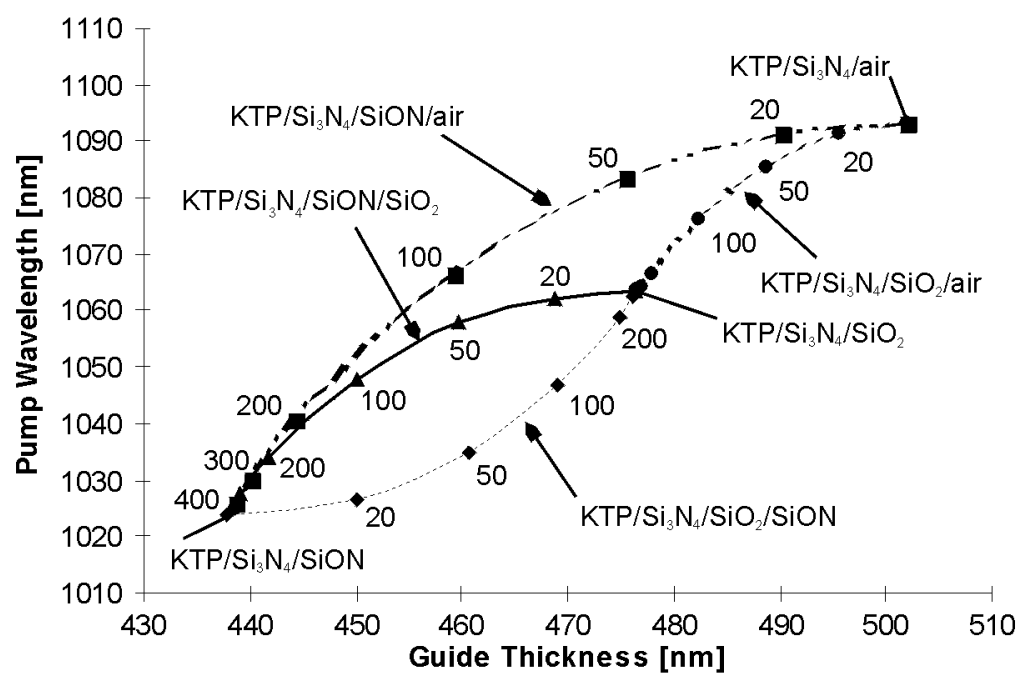

Fig. 2. Peak positions of $\mathrm{KTP} / \mathrm{Si}_{3} \mathrm{~N}_{4}$ / "cladding" devices.

Figure 2 shows the general behaviour of the peak positions of the KTP/ $\mathrm{Si}_{3} \mathrm{~N}_{4}$ / "cladding" device. Particular claddings consist of the following combinations: $\mathrm{SiON} /$ air, $\mathrm{SiO}_{2}$ /air, $\mathrm{SiON} / \mathrm{SiO}_{2}$, and $\mathrm{SiO}_{2} / \mathrm{SiON}$, respectively. It is quite clear that the points of intersection of the curves belong to the peak positions of three-layer devices, as depicted in this figure. Combining the claddings described 
above, this silicon cladding based structure may efficiently convert wavelengths from about $1025 \mathrm{~nm}$ to $1094 \mathrm{~nm}$. However, each particular combination follows those strict rules concerning the peak FWHM described above. Thus, having any cladding of arbitrary refractive index, one can design a structure efficiently converting any wavelength within the region determined by the lowest and the highest refractive index of the cladding, respectively.

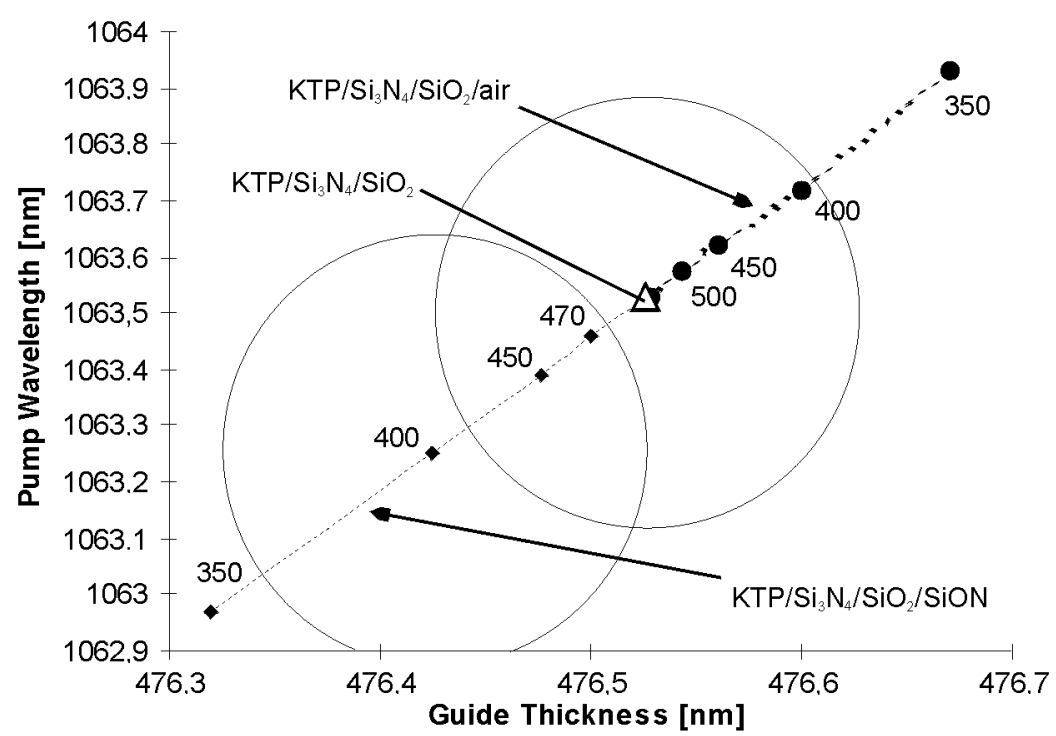

Fig. 3. A detail of peak positions of the $\mathrm{KTP} / \mathrm{Si}_{3} N_{4}$ / "cladding" device at the vicinity of the $\mathrm{SiO}_{2}$ cladding. As an example, the two circles indicate the FWHM of the peaked efficiency for the peak for the three-layer device $\mathrm{KTP} / \mathrm{Si}_{3} \mathrm{~N}_{4} / \mathrm{SiO}_{2}$ (right-upper circle) and for the device $\mathrm{KTP} / \mathrm{Si}_{3} \mathrm{~N}_{4} / \mathrm{SiO}_{2}(w=400 \mathrm{~nm}) / \mathrm{SiON}$.

Let us study the points of intersections and their close vicinity in detail. Figure 3 shows the peak positions at the vicinity of the $\mathrm{SiO}_{2}$ cladding (namely $\mathrm{KTP} / \mathrm{Si}_{3} \mathrm{~N}_{4} / \mathrm{SiO}_{2} /$ air and $\mathrm{KTP} / \mathrm{Si}_{3} \mathrm{~N}_{4} / \mathrm{SiO}_{2} / \mathrm{SiON}$ ). As it is seen from this figure, the peak position is fairly tolerant for changes of the idler layer thickness and even more for changes of the outermost cladding layer (superstrate) at all. One may thus conclude that a device having a guide thickness approximately equal to a required one for a given three-layer waveguide can be finally tuned (to reach the maximum conversion efficiency) by a suitable combination of relatively thick idler layer and an outermost layer. The idler layer must consist of the same material as the cladding of the three-layer waveguide considered before. Similar study for $\mathrm{SiON}$ idler layer is shown in Fig. 4. It can be seen again that the peak positions exhibit tolerant dependence with respect to the outermost layer material. On the contrary, Fig. 5 shows the case, where the $\mathrm{SiO}_{2}$ /air and the $\mathrm{SiON} /$ air claddings were considered. It is thus quite clear that such claddings exhibit fairly different behaviour without any possibility to ease reaching of the peak. 


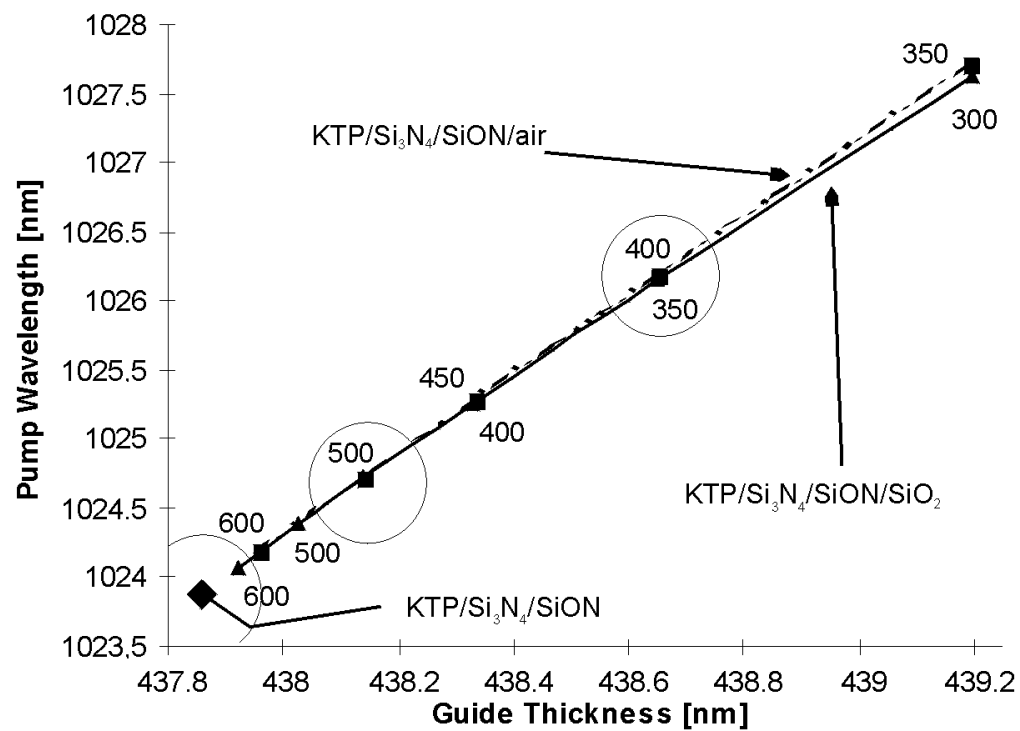

Fig. 4. A detail of peak positions of $\mathrm{KTP} / \mathrm{Si}_{3} \mathrm{~N}_{4} /$ "cladding" device at the vicinity of the SiON cladding (those three circles schematically indicate the efficiency peak FWHM).

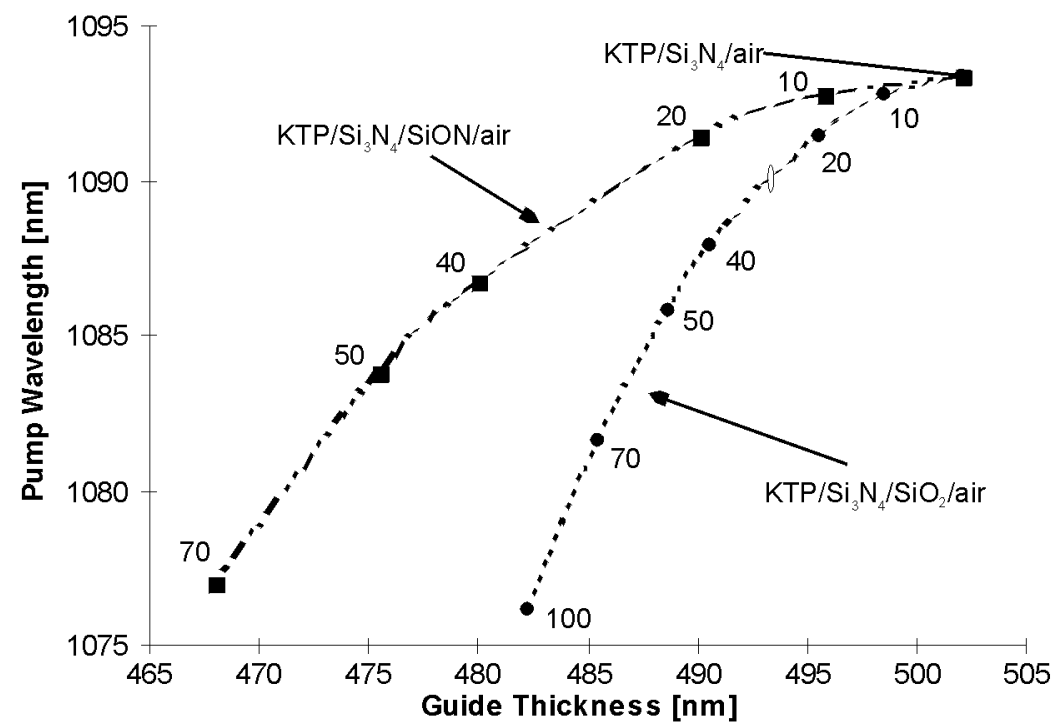

Fig. 5. A detail of peak positions of the $\mathrm{KTP} / \mathrm{Si}_{3} \mathrm{~N}_{4} /$ "cladding" device at the vicinity of the air cladding (assumed $\mathrm{SiON} /$ air cladding and $\mathrm{SiO}_{2}$ /air one for small idler thicknesses). (A small ellipse indicates the FWHM of the peak.).

An analogous analysis as done in Fig. 2 may be performed for the guiding layer, taking into account that only particular values of the refractive indices are technically a vailable. Figure 6 shows the general behaviour of the peak positions for different guiding layers. Namely, we study here the $\mathrm{KTP} / \mathrm{Si}_{3} \mathrm{~N}_{4} / \mathrm{Y}_{2} \mathrm{O}_{3}$ /"cladding" 


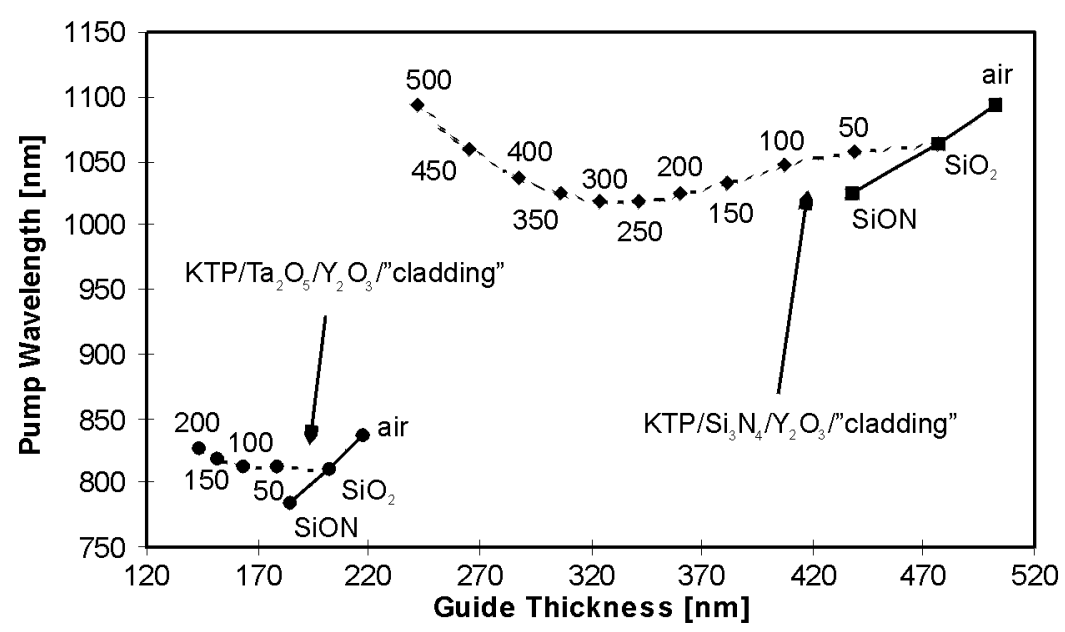

Fig. 6. The comparison of peak positions of two four layer-waveguides having different guiding layer $\left(\mathrm{KTP} / \mathrm{Si}_{3} \mathrm{~N}_{4} / \mathrm{Y}_{2} \mathrm{O}_{3}\right.$ / "cladding" and $\mathrm{KTP} / \mathrm{Ta}_{2} \mathrm{O}_{5} / \mathrm{Y}_{2} \mathrm{O}_{3}$ / "cladding" devices).

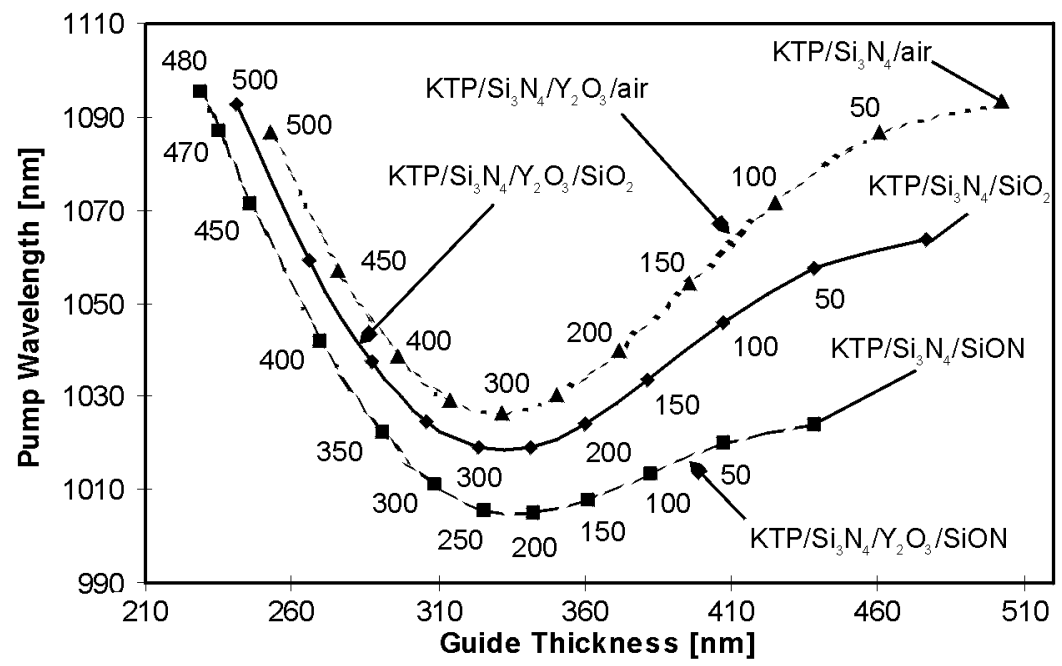

Fig. 7. A detailed study of the $\mathrm{KTP} / \mathrm{Si}_{3} \mathrm{~N}_{4} / \mathrm{Y}_{2} \mathrm{O}_{3} /$ "cladding" device.

$\left(\mathrm{Y}_{2} \mathrm{O}_{3} ; n \approx 1.92\right)$ and the $\mathrm{KTP} / \mathrm{Ta}_{2} \mathrm{O}_{5} / \mathrm{Y}_{2} \mathrm{O}_{3} /$ "cladding" devices ( $\mathrm{Ta}_{2} \mathrm{O}_{5}$; $n \approx 2.1$ ). According to Fig. 6 , it can be said that the higher the refractive index of the guiding layer is, the shorter wavelength is efficiently converted and the thinner guiding layer is needed. The study of the idler layer influence on the peak tuning is in fact analogous (see also [10]). Figure 7 shows the detail of the peak position behaviour of the $\mathrm{KTP} / \mathrm{Si}_{3} \mathrm{~N}_{4} / \mathrm{Y}_{2} \mathrm{O}_{3}$ / "cladding" devices (detailed description of the particular cladding is schematically provided inside the figure). One may thus perform a similar treatment, but now for a five layer-waveguide (e.g. $\mathrm{KTP} / \mathrm{Si}_{3} \mathrm{~N}_{4} / \mathrm{Y}_{2} \mathrm{O}_{3} / \mathrm{SiON} /$ air and that like), as was done through Figs. 2, 3, 4, 


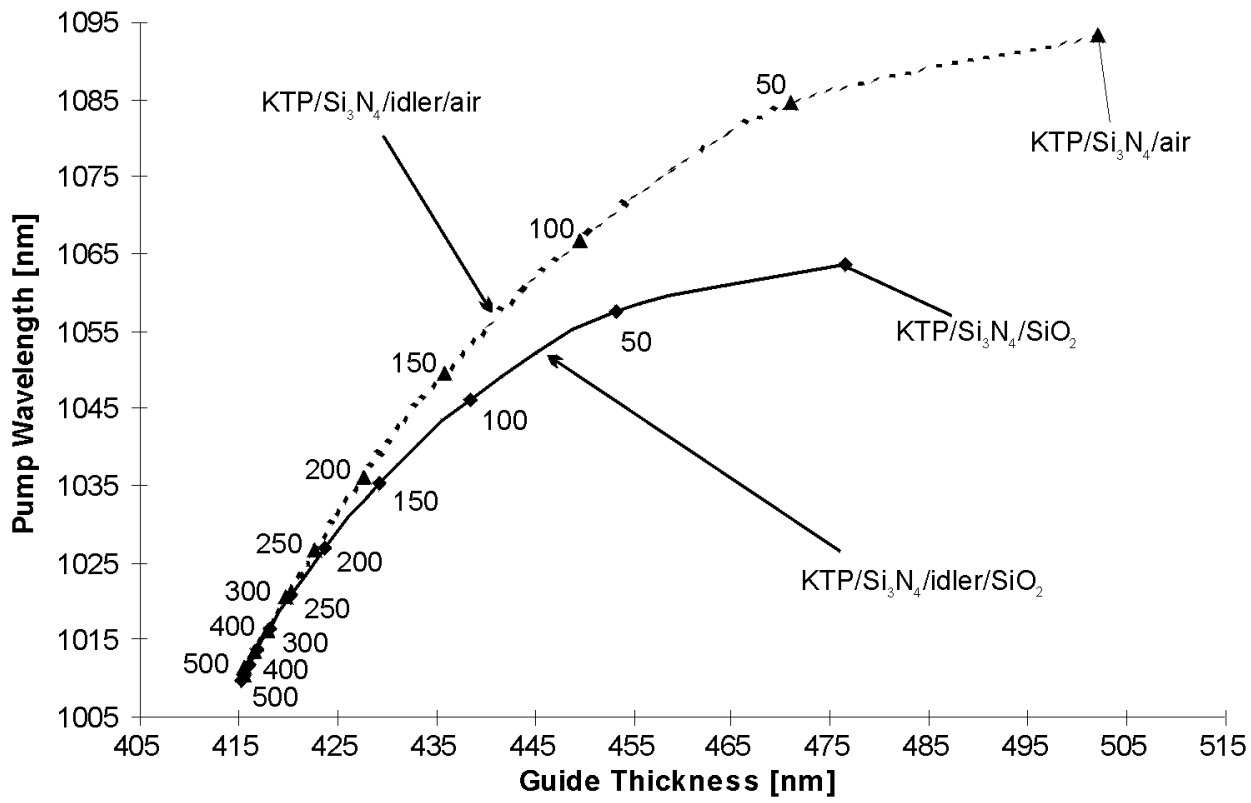

Fig. 8. Peak positions of the device $\mathrm{KTP} / \mathrm{Si}_{3} \mathrm{~N}_{4} /$ fictitious idler/ "cladding".

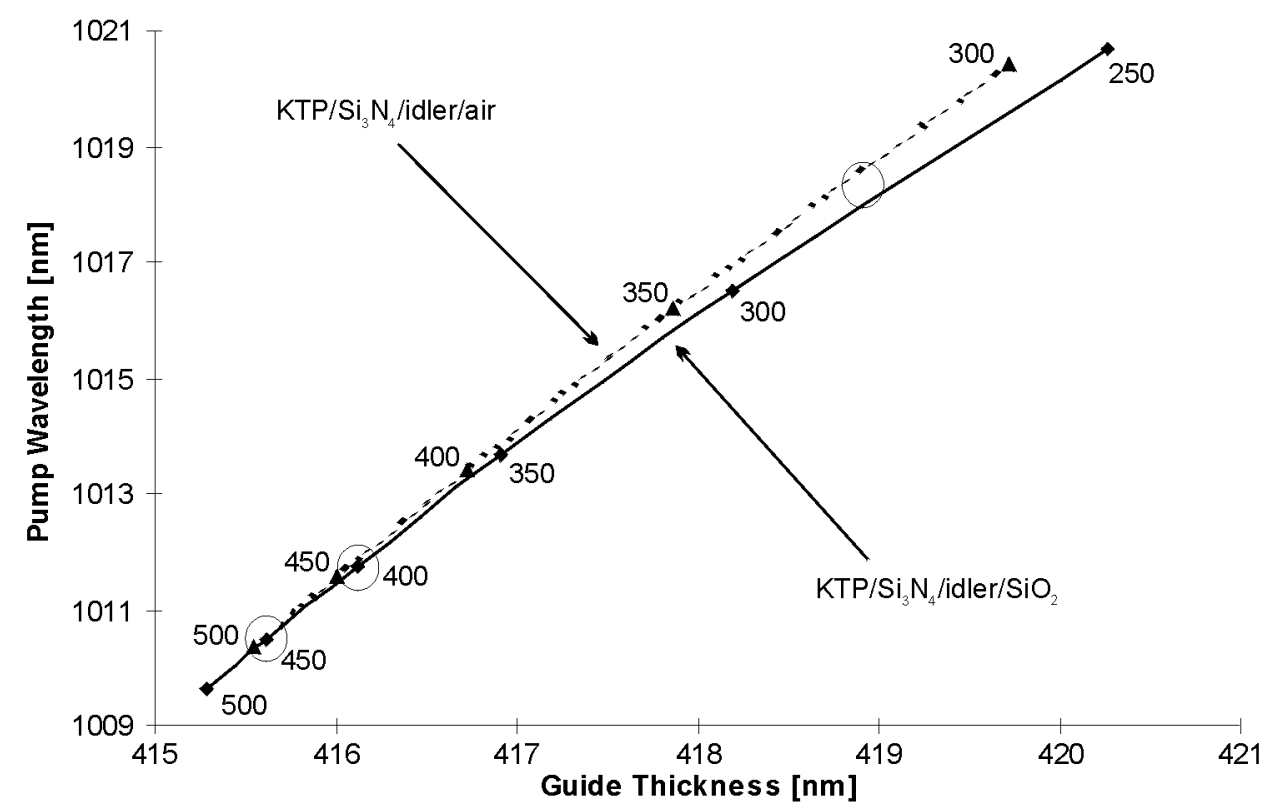

Fig. 9. A detail of the peak behaviour of the $\mathrm{KTP} / \mathrm{Si}_{3} \mathrm{~N}_{4}$ /fictitious idler/ "cladding" for essentially thick (hundreds $\mathrm{nm}$ ) idler layer $\left(\mathrm{Y}_{2} \mathrm{O}_{3}\right.$ ). (Those three circles schematically indicate the efficiency peak FWHM.). 
and 5, respectively. This may be also simply done by a suitable combination of the results and thoughts given above. Thus, we shall omit this study for the sake of brevity.

However, presented studies gave just particular solutions of the less critical ways to reach the efficiency peak. Let us remind the principal condition (8) yielding the Cerenkov peak. To reach the less critical behaviour of the structure with respect to the efficiency peak, we need to have a structure with a required effective refractive index. Hence, introducing the idler layer of the refractive index slightly above the required value of the refractive index seems to be very promising for practical applications. For example, the waveguides $\mathrm{KTP} / \mathrm{Si}_{3} \mathrm{~N}_{4} /$ "fictitious idler" $/ \mathrm{SiO}_{2}$ and $\mathrm{KTP} / \mathrm{Si}_{3} \mathrm{~N}_{4}$ / "fictitious idler"/air, respectively, behave as shown in Fig. 8 and in Fig. 9 (detail). The refractive index of the fictitious idler was chosen as $n_{\text {fict }}=n_{\mathrm{Y} 2 \mathrm{O} 3}-0.13 \approx 1.8$. The structure with such an idler layer of the thickness of about 350 or $500 \mathrm{~nm}$ exhibits lower sensitivity of the efficiency to the pump wavelength changes similar to those as were discussed through Fig. 3 and Fig. 4, respectively. Moreover, we are not further limited by a given peak position, but we may expect - in general - some tunability with respect to a chosen pump wavelength by introducing a suitable material of requested refractive index. For practical implementation, a suitable material such as $\mathrm{SiO}_{x} \mathrm{~N}_{y}$ can be used for an idler layer.

\section{Conclusions}

This paper theoretically studied the C̆SHG conversion efficiency peak behaviour as a function of a material composition of the four-layer waveguide. It was shown that some specific material compositions offer a less critical way to reach the efficiency peak. Firstly, the structure may be fine-tuned with respect to a given superstrate material by a combination of that material as the idler layer and some other one as the outermost layer. Secondly, the idler layer of the refractive index slightly above the required value of the effective index may essentially relax the demands for reaching the peak. Finally, a suitable material combination may shift, otherwise fixed, peak position. While the guide thickness tolerance lower than $0.5 \mathrm{~nm}$ is required for a simple three-layer waveguide structure, the idler layer inaccuracy of about $10 \mathrm{~nm}$ can be tolerated if combined with a fine tuning of the pump wavelength, which is rather promising for reaching of the efficiency peak experimentally.

\section{References}

[1] E.M. Conwell, IEEE J. Quantum Electron. QE-9, 867 (1973).

[2] A. Yariv, IEEE J. Quantum Electron. QE-9, 919 (1973).

[3] H. Tamada, IEEE J. Quantum Electron. 27, 502 (1991).

[4] J. Čtyroký, L. Kotačka, Opt. Quantum Electron. 32, 799 (2000).

[5] L. Kotačka, J. Ctyroký, submitted to Opt. Quantum Electron. 
[6] N. Asai, H. Tamada, I. Fujiwara, J. Seto, J. Appl. Phys. 72, 4521 (1992).

[7] T. Doumuki, H. Tamada, M. Saitoh, Appl. Phys. Lett. 64, 3533 (1994).

[8] T. Doumuki, H. Tamada, M. Saitoh, Appl. Phys. Lett. 65, 2519 (1994).

[9] R.-S. Chang, R.-Y. Shaw, J. Mod. Opt. 45, 103 (1998).

[10] L. Kotačka, J. Čtyroký, H.J.W.M. Hoekstra, in: Non-linear Guided Waves and Their Applications, OSA Tech. Digest, Dijon 1999, OSA, Washington D.C. 1999, p. 380 .

[11] C. Tsao, Optical Fibre Waveguide Analysis, Oxford University Press, Oxford 1992.

[12] M. Born, E. Wolf, Principles of Optics, 6th ed., Pergamon Press, Oxford 1993. 